\title{
Unscented Transformation of Vehicle States in SLAM
}

\author{
Juan Andrade-Cetto, Teresa Vidal-Calleja, and Alberto Sanfeliu \\ Institut de Robòtica i Informàtica Industrial, UPC-CSIC \\ Llorens Artigas 4-6, Barcelona, 08028 Spain \\ \{cetto, tvidal, sanfeliu\}@iri.upc.es
}

\begin{abstract}
In this article we propose an algorithm to reduce the effects caused by linearization in the typical EKF approach to SLAM. The technique consists in computing the vehicle prior using an Unscented Transformation. The UT allows a better nonlinear mean and variance estimation than the EKF. There is no need however in using the UT for the entire vehiclemap state, given the linearity in the map part of the model. By applying the UT only to the vehicle states we get more accurate covariance estimates. The a posteriori estimation is made using a fully observable EKF step, thus preserving the same computational complexity as the EKF with sequential innovation. Experiments over a standard SLAM data set show the behavior of the algorithm.
\end{abstract}

\section{INTRODUCTION}

Simultaneous localization and map building (SLAM) is the problem of localizing a robot from landmark observations, while at the same time, incrementally building a map of these landmarks [1]. The problem can be casted as a state estimation problem, with the Extended Kalman Filter (EKF) the most widely accepted tool for solving it [2], [3]. One drawback however with the use of the EKF, is in the linear propagation of means and covariances. Vehicle and sensor models are usually of a very high nonlinear nature, and the effects of linearization required in the EKF can lead to filter divergence [4].

This situation has prompted the use of particle filters for a non parametric approximation of vehicle and map probability density functions in SLAM. Particle filters approximate the state space by random sampling the posterior distribution, and may require many samples to accurately model the nonlinear effects in both vehicle and measurement models. A middle ground is to use a deterministic approach for the nonlinear propagation of means and covariances. One such solution is the use of the Unscented Kalman Filter (UKF) [5], [6]. An unscented transformation is similar to a particle filter in that it samples the $p d f$, but instead of doing it randomly, a careful selection of deterministic sigma points is made so as to preserve the moments of the distribution.

Deterministically choosing the particles is a computationally efficient solution for the nonlinear propagation of means and covariances, but doing so for the full state vector in SLAM may not be appropriate. There is no need to use particles in the computation of the map prior, given its linear nature. Thus, by

This work is supported by the Spanish Council of Science and Technology under projects DPI 2001-2223, and DPI 2004-5414. using the Unscented Transformation (UT) only for the vehicle states we are able to reduce the computational complexity (compared to a full UKF), and to produce, at the same time, tighter covariance estimates.

The remaining of the article is structured as follows. In Section II, the original EKF approach to SLAM is briefly reviewed, and the necessary notational conventions are made. In Section III, the UKF is explained, detailing the consequences of nonlinearly propagating the entire vehicle and map state vector, as opposed to only propagating the vehicle states. Section IV is devoted to a numerical comparison of the three approaches: EKF, UKF, and vehicle only UT. Finally, Section $\mathrm{V}$ contains some concluding remarks.

\section{Problem Statement}

The benchmark problem of SLAM is an example of high order nonlinear system estimation, where most of the states do not vary. The structure of a joint mobile robot-landmark model is as follows. The state vector $\mathbf{x}_{k}$ contains the pose of the robot $\mathbf{x}_{r, k}$ at time step $k$, and a vector of map features $\mathbf{x}_{f}$ (note the lack of time dependency for $\mathbf{x}_{f}$, meaning that the map features are stationary). In the EKF approach, the posterior is represented by a multivariate Gaussian distribution over the state $\mathbf{x}_{k}$, with mean and covariance state estimates

$$
\begin{gathered}
\mathbf{x}_{k \mid k}=\left[\begin{array}{l}
\mathbf{x}_{r, k \mid k} \\
\mathbf{x}_{f, k \mid k}
\end{array}\right] \\
\mathbf{P}_{k \mid k}=\left[\begin{array}{cc}
\mathbf{P}_{r, k \mid k} & \mathbf{P}_{r f, k \mid k} \\
\mathbf{P}_{r f, k \mid k}^{\top} & \mathbf{P}_{f, k \mid k}^{\top}
\end{array}\right]
\end{gathered}
$$

where $\mathbf{P}_{r, k \mid k}$ is the covariance of the robot pose estimate, $\mathbf{P}_{f, k \mid k}$ is the covariance of the position estimate for the entire map, and $\mathbf{P}_{r f, k \mid k}$ is the cross-covariance between the robot and landmark estimates.

The motion of the robot and the measurement of the map features are governed by the nonlinear discrete-time state transition model

$$
\begin{aligned}
\mathbf{x}_{r, k+1} & =\mathbf{f}\left(\mathbf{x}_{r, k}, \mathbf{u}_{k}, \mathbf{v}_{k}\right) \\
\mathbf{z}_{k} & =\mathbf{h}\left(\mathbf{x}_{k}\right)+\mathbf{w}_{k}
\end{aligned}
$$

in which the input vector $\mathbf{u}_{k}$ is the vehicle control command, and $\mathbf{v}_{k}$ and $\mathbf{w}_{k}$ are typically modeled as Gaussian random vectors with zero mean and covariance matrices $\mathbf{Q}$ and $\mathbf{R}$, respectively, representing on the one side unmodeled robot 
dynamics and system noise; and measurement noise on the other side.

Provided the set of observations $Z^{k}=\left\{\mathbf{z}_{1}, \ldots, \mathbf{z}_{k}\right\}$ was available for the computation of the current map estimate $\mathbf{x}_{k \mid k}$, the expression

$$
\mathbf{x}_{k+1 \mid k}=\mathbf{f}\left(\mathbf{x}_{k \mid k}, \mathbf{u}_{k}, \mathbf{0}\right)
$$

gives an a priori noise-free estimate of the new locations of the robot and map features after the vehicle control command $\mathbf{u}_{k}$ is input to the system. Similarly,

$$
\mathbf{z}_{k+1 \mid k}=\mathbf{h}\left(\mathbf{x}_{k+1 \mid k}, \mathbf{0}\right)
$$

constitutes a noise-free a priori estimate of sensor measurements.

Given that the landmarks are considered stationary, their a priori estimate is simply $\mathbf{x}_{f, k+1 \mid k}=\mathbf{x}_{f, k \mid k}$; and the a priori estimate of the map state error covariance showing the increase in robot and landmark localization uncertainty is given by

$$
\mathbf{P}_{k+1 \mid k}=E\left[\tilde{\mathbf{x}}_{k+1 \mid k} \tilde{\mathbf{x}}_{k+1 \mid k}^{\top}\right]=\mathbf{F P}_{k \mid k} \mathbf{F}^{\top}+\mathbf{G Q G}^{\top}
$$

The Jacobian matrices $\mathbf{F}$ and $\mathbf{G}$ contain the partial derivatives of $\mathbf{f}$ with respect to $\mathbf{x}$ and $\mathbf{v}$ respectively, evaluated at $\left(\mathbf{x}_{k \mid k}, \mathbf{u}_{k}, \mathbf{0}\right)$.

Assuming that a new set of landmark observations $\mathbf{z}_{k+1}$ coming from sensor data has been correctly matched to their map counterparts, one can compute the innovation (the error between the measurements and the estimates) as $\tilde{\mathbf{z}}_{k+1 \mid k}=$ $\mathbf{z}_{k+1}-\mathbf{z}_{k+1 \mid k}$.

This error aids in revising the map and robot locations. The a posteriori state estimate is

$$
\mathbf{x}_{k+1 \mid k+1}=\mathbf{x}_{k+1 \mid k}+\mathbf{K} \tilde{\mathbf{z}}_{k+1 \mid k}
$$

and the Kalman gain is computed with

$$
\mathbf{K}=\mathbf{P}_{k+1 \mid k} \mathbf{H}^{\top} \mathbf{S}^{-1}
$$

where $\mathbf{S}$ is termed the measurement innovation matrix,

$$
\mathbf{S}=\mathbf{H P}_{k+1 \mid k} \mathbf{H}^{\top}+\mathbf{R}
$$

and $\mathbf{H}$ contains the partial derivatives of $\mathbf{h}$ with respect to $\mathbf{x}$ evaluated at $\left(\mathbf{x}_{k+1 \mid k}, \mathbf{0}\right)$.

Finally, the a posteriori estimate of the map state error covariance must also be revised once a measurement has taken place. It is revised with the Joseph form to guarantee positive semi-definiteness.

$$
\mathbf{P}_{k+1 \mid k+1}=(\mathbf{I}-\mathbf{K H}) \mathbf{P}_{k+1 \mid k}(\mathbf{I}-\mathbf{K H})^{\top}+\mathbf{K R K}^{\top}
$$

Overall, the computational complexity of the EKF is $O\left(n^{3}\right)$, with $n$ the number of landmarks in the map. The inverse required in (9) sets this complexity bound, but when sequential innovation is used, it can be further reduced to $O\left(n^{2}\right)$.

\section{Nonlinear Propagation of State Estimates}

Julier and Uhlmann [6]-[8] introduced a filter called the Unscented Kalman Filter (UKF) founded on the intuition that it is easier to approximate a Gaussian distribution than to approximate nonlinear functions. The UKF not only leads to more accurate results than the EKF, but also, it generates better estimates of the state covariances. The reason, the UKF approximates the posterior mean and covariance accurately to the 2nd order for any nonlinearity, in contrast with the EKF which only takes into account the first order term of a Taylor series during linearization.

\section{A. $U K F$}

The Unscented Transform (UT) forms the core of the UKF algorithm, it consists in choosing a set $\mathcal{S}$ of points (sigma points $\mathcal{X}^{i}$ and weights $W^{i}$ ) so that their mean and covariance are $\mathbf{x}$ and $\mathbf{P}$. The nonlinear model in (4) is applied to each point, and the weighted statistics of the transformed points form an estimate of the nonlinearly transformed mean and covariance.

The UKF algorithm is similar in structure to the EKF algorithm. Once the sigma points are obtained (see Appendix I), the a priori estimates are evaluated with

$$
\begin{gathered}
\mathcal{X}_{k+1 \mid k}^{i}=\mathbf{f}\left(\mathcal{X}_{k \mid k}^{i}, \mathbf{u}_{k}, \mathcal{V}_{k}^{i}\right) \\
\mathbf{x}_{k+1 \mid k}=\sum_{i=0}^{p} W^{i} \mathcal{X}_{k+1 \mid k}^{i} \\
\mathbf{P}_{k+1 \mid k}=\sum_{i=0}^{p} W^{i}\left(\mathcal{X}_{k+1 \mid k}^{i}-\mathbf{x}_{k+1 \mid k}\right)\left(\mathcal{X}_{k+1 \mid k}^{i}-\mathbf{x}_{k+1 \mid k}\right)^{\top}
\end{gathered}
$$

In the UKF the innovations are

$$
\begin{gathered}
\mathcal{Z}_{k+1 \mid k}^{i}=\mathbf{h}\left(\mathcal{X}_{k+1 \mid k}^{i}\right)+\mathcal{W}_{k}^{i} \\
\mathbf{z}_{k+1 \mid k}=\sum_{i=0}^{p} W^{i} \mathcal{Z}_{k+1 \mid k}^{i}
\end{gathered}
$$

$\mathbf{P}_{k+1 \mid k}^{z z}=\sum_{i=0}^{p} W^{i}\left(\mathcal{Z}_{k+1 \mid k}^{i}-\mathbf{z}_{k+1 \mid k}\right)\left(\mathcal{Z}_{k+1 \mid k}^{i}-\mathbf{z}_{k+1 \mid k}\right)^{\top}$

And the a posteriori estimates are

$$
\begin{gathered}
\mathbf{P}_{k+1 \mid k}^{x z}=\sum_{i=0}^{p} W^{i}\left(\mathcal{X}_{k+1 \mid k}^{i}-\mathbf{x}_{k+1 \mid k}\right)\left(\mathcal{Z}_{k+1 \mid k}^{i}-\mathbf{z}_{k+1 \mid k}\right)^{\top} \\
\mathbf{K}=\mathbf{P}_{k+1 \mid k}^{x z}\left(\mathbf{P}^{z z}\right)^{-1} \\
\mathbf{x}_{k+1 \mid k+1}=\mathbf{x}_{k+1 \mid k}+\mathbf{K} \tilde{\mathbf{z}}_{k+1 \mid k} \\
\mathbf{P}_{k+1 \mid k+1}=\mathbf{P}_{k+1 \mid k}-\mathbf{K} \mathbf{P}^{z z} \mathbf{K}^{\top}
\end{gathered}
$$

The terms $\mathcal{V}^{i}$ and $\mathcal{W}^{i}$ are sigma points for the noise terms. To compute them, an augmented point set is built from $\mathbf{x}, \mathbf{P}, \mathbf{Q}$ and $\mathbf{R}$. See [6] for details.

The update estimation in the UKF algorithm requires an augmented sigma point set for the entire state vector in (17). The computation of these points requires a Cholesky factorization of $\mathbf{P}$, with computational complexity $O\left(n^{3}\right)$. 


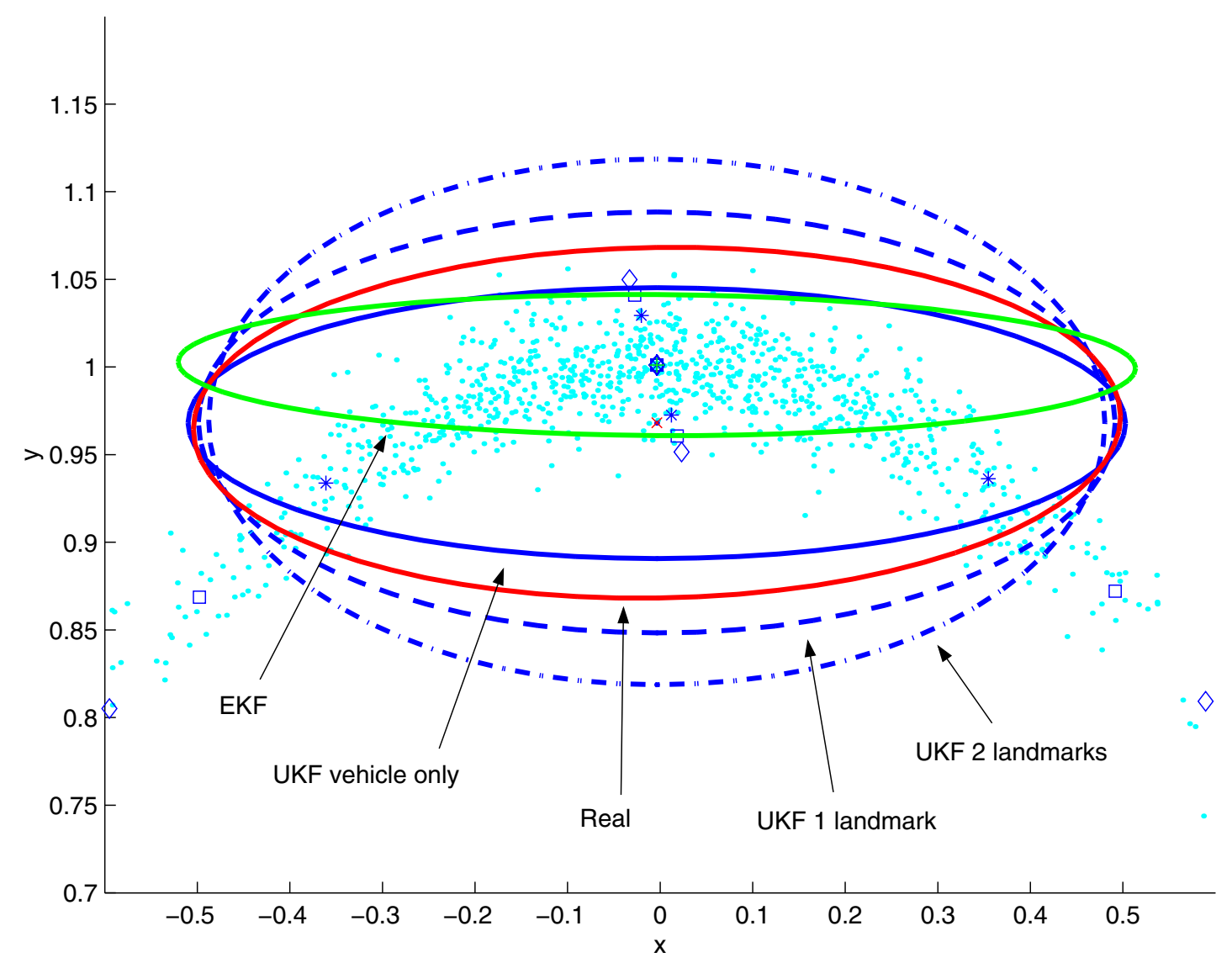

Fig. 1. Unscented transformation of the augmented state vector produces more $\sigma$ points that project to the mean. To preserve the statistics, the remaining $\sigma$ points are pushed away from the mean, thus producing more conservative estimates of the covariance in the nonlinear case. In red (x): actual mean and covariance; in green $(+)$ : linear transformation of mean and covariance; in blue $(*)$ : nonlinear transformation of mean and covariance for robot only; in blue dashed $(\square)$ : nonlinear transformation for robot and one landmark; in blue dash dot $(\diamond)$ : nonlinear transformation for robot and 2 landmarks.

\section{B. UT of Vehicle States}

In SLAM, the dimension of the state vector is proportional to the number of landmarks in the map, and every time a new feature is added to the map, the state vector is also augmented. Moreover, when the UKF is used, the required number of sigma points is also proportional to the number of landmarks in the map. These sigma points are symmetrically distributed along the hyperellipsoid representing the covariance of the entire state space. Now, every time a new landmark is added, the corresponding sigma points will map the new state space directions, and project to the mean on the vehicle space hyperplane, with the rest of the points being scaled accordingly, in order to preserve the first and second order statistics of the entire distribution, i.e., $E[\tilde{\mathbf{x}}]$ and $E\left[\tilde{\mathbf{x}} \tilde{\mathbf{x}}^{\top}\right]$.

Looking at the projected hyperellipsoid representing the vehicle covariance we see that as the number of landmarks increases, the UT maintains the true mean of the vehicle prior, but it underestimates the vehicle covariance. Figure 1 shows this situation. In the plot, a typical nonlinear motion model of one meter with translational variance of $2 \mathrm{~cm}$ and rotational variance of $15^{\circ}$ is sampled 1000 times (cyan dots). The true mean and $2 \sigma$ hyperellipsoid for the true covariance are also plotted (in red). The linear transformation of the original $p d f$ (the prior step of the EKF) is shown in green. In the plot, the mean computed by the EKF is slightly above the true mean, and the linear transformation of the vehicle covariance is largely overestimated. Now, the UT for this nolandmark model is at the true mean, with also an overestimated covariance, but not as much as the linear transformation (the mean and corresponding 5 sigma points are plotted as blue stars, and the $2 \sigma$ covariance hyperellipsoid is plotted as a continuous blue line. Adding one two-dimensional landmark to the map at $(0,0)$ and with variance $1 \mathrm{~cm}$, the required number of sigma points increases from 5 to 9 , and their projection onto the Cartesian vehicle coordinates is shown as blue boxes. Notice how the new sigma point locations emulate the previous set, but are pushed away from the mean (scaled), with more points located precisely at the mean. The corresponding covariance hyperellipsoid projection (dashed blue) is slightly underestimated with respect to the previous UT and the original covariance. Finally, adding one more landmark to the map, the required number of sigma points is 13 , and their projection onto the vehicle state space is shown as blue diamonds, with the corresponding $2 \sigma$ covariance hyperellipsoid shown in dash 


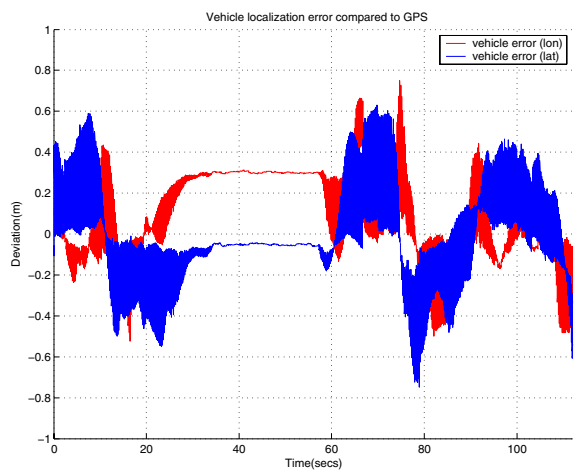

(a) EKF Vehicle error

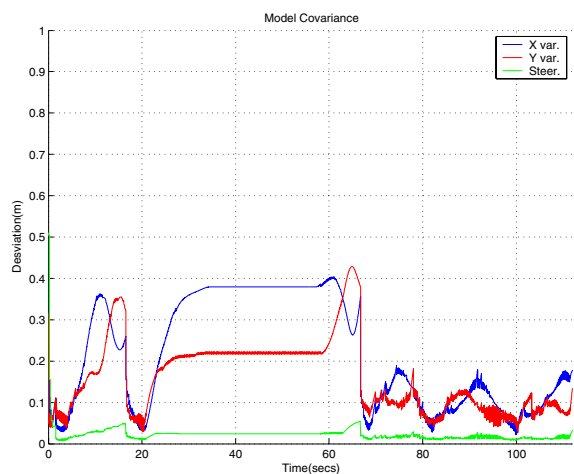

(d) EKF Vehicle covariance

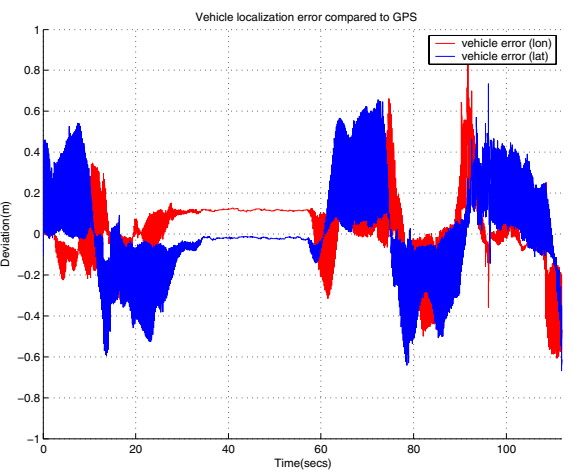

(b) UKF Vehicle error

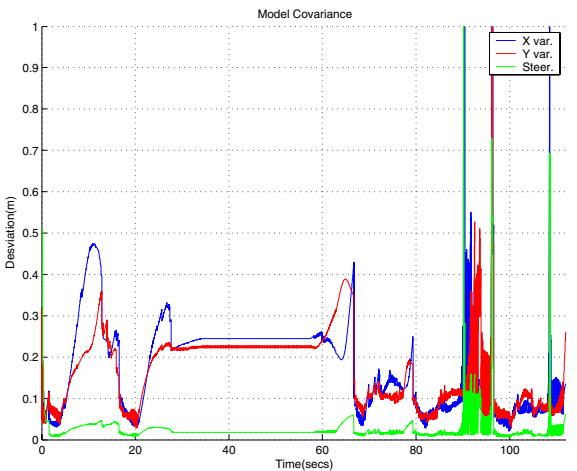

(e) UKF Vehicle covariance

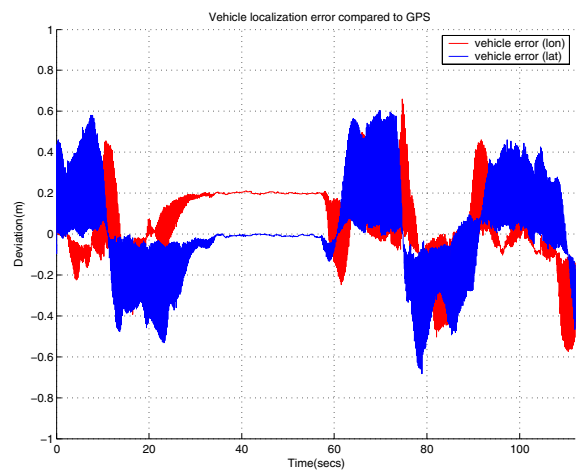

(c) UT Vehicle error

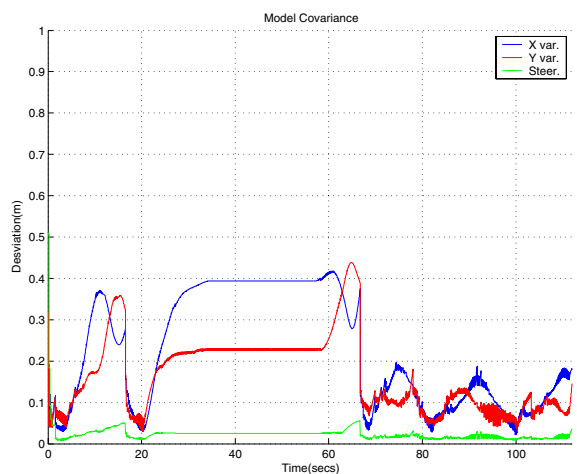

(f) UT Vehicle covariance

Fig. 2. Comparison of the Extended Kalman Filter, the Unscented Kalman Filter, and Unscented Transformation of Vehicle States only on the Car Park dataset from the University of Sydney. The Unscented Transformation of Vehicle states is not only computationally more efficient than the full UKF, but avoids data association errors due to covariance overestimation in the full UKF.

dot blue. Even when the computed mean is equal to the one computed in the previous two cases, the sigma points are further pushed away from the mean, and the corresponding covariance is also underestimated. The conclusion is that when the number of landmarks increases significantly, the UT is still good at computing the nonlinear mean estimate for the vehicle position, but it largely underestimates its covariance.

The variance estimates of the dynamic states (a priori vehicle location estimation) should not depend on the number of static states on the model (landmarks), but only on the characteristics of the motion model. The map entries being static have a linear model (identity in fact), and no UT is necessary for this transformation. The underestimation of the covariance is the result of projecting a higher dimensional hyperellipsoid of such transformation onto the vehicle states hyperplane. For this reason we propose to apply the UT only to the vehicle states, and not to the full state vector during the prediction step.

The plant Jacobian matrices in (6) can be decomposed into two block diagonal matrices, explicitly differentiating vehicle derivatives $\mathbf{F}_{r}$ and $\mathbf{G}_{r}$. Equation (6) can be rewritten as

$$
\mathbf{P}_{k+1 \mid k}=\left[\begin{array}{cc}
\mathbf{F}_{r} \mathbf{P}_{r, k \mid k} \mathbf{F}_{r}^{\top}+\mathbf{G}_{r} \mathbf{Q} \mathbf{G}_{r}^{\top} & \mathbf{F}_{r} \mathbf{P}_{r f, k \mid k} \\
\left(\mathbf{F}_{r} \mathbf{P}_{r f, k \mid k}\right)^{\top} & \mathbf{P}_{f, k \mid k}
\end{array}\right]
$$

Our algorithm substitutes the a priori computation of the vehicle covariance in the EKF, with the one computed using the UT; while preserving the rest of the covariance matrix. That is, the upper left submatrix is substituted with

$$
\begin{gathered}
\mathbf{P}_{r, k+1 \mid k}^{*}=\mathbf{P}_{r, k+1 \mid k}+\Delta \\
=\sum_{i=0}^{p} W^{i}\left(\mathcal{X}_{r, k+1 \mid k}^{i}-\mathbf{x}_{r, k+1 \mid k}\right)\left(\mathcal{X}_{r, k+1 \mid k}^{i}-\mathbf{x}_{r, k+1 \mid k}\right)^{\top}
\end{gathered}
$$

We have seen empirically that by substituting the submatrix $\mathbf{P}_{r, k+1 \mid k}$ with $\mathbf{P}_{r, k+1 \mid k}^{*}$ in (21), the $p s d$ properties of $\mathbf{P}_{k+1 \mid k}^{*}$ still hold. That is, by underestimating $\mathbf{P}_{r}^{*}$ with the UT, and still computing the rest of Jacobians in $\mathbf{P}_{k+1 \mid k}^{*}$ with the linear EKF, the condition

$$
\mathbf{P}_{k+1 \mid k}^{*}=\left[\begin{array}{cc}
\mathbf{P}_{r, k+1 \mid k}+\Delta & \mathbf{P}_{r f, k+1 \mid k} \\
\mathbf{P}_{r f, k+1 \mid k}^{\top} & \mathbf{P}_{f, k+1 \mid k}
\end{array}\right] \geq 0
$$

still holds.

\section{EXPERIMENTAL RESULTS. EKF, UKF, AND VEHICLE-ONLY UT}

We use the University of Sydney Car Park dataset [9] to show estimation results when the prediction is made with the nonlinear approach proposed in this article, and compare them with both an EKF, and an UKF. With the proposed technique, we have been able to reduce the bias caused by linearization of the nonlinear plant model in the EKF. 
The nonlinear vehicle and measurement models used in our experiments are similar to those in [10], and are shown in Appendix II for clarity. The only substantial difference is in the use of an anchor feature not under estimation in the measurement model, in order to guarantees full observability [11], [12].

Figures 2 and 3 contain plots comparing the three algorithms, for the vehicle location error, the vehicle location variance, and the final map, respectively. The first column of plots in Figure 2 corresponds to a fully observable Extended Kalman Filter, in which the first observed landmark (located at coordinates $(2.8953,-4.0353)$ ) is used as a global anchor. The second column of plots corresponds to a full implementation of the Unscented Kalman Filter. The last column of plots corresponds to our hybrid implementation: using the Unscented Transformation for a nonlinear approximation of vehicle priors, and filling the rest of the a priori covariance estimate with the required vehicle to landmark Jacobians, plus the typical a posteriori computations in the EKF. This technique is not only computationally more efficient (it requires on the one hand a small and fixed number of sigma points in the computation of the UT, 7 in our case, and also, it can make efficient use of sequential innovation as in the traditional EKF approach to SLAM), but reduces significantly the errors made during linearization in the computation of the vehicle priors.

In congruence with our conclusions from Section III, the Unscented Kalman Filter, which utilizes the entire state vector in the computation of the sigma point set, ends up underestimating the vehicle localization covariance at the beginning of the experiment. This can be appreciated in Figure 2e. However, once observations are made, the algorithm makes a good job at reducing the overall vehicle covariances. The vehicle location error estimate is also slightly smaller for the entire UKF approach, compared to the other two algorithms. See frame $\mathrm{b}$ in Figure 2.

Unfortunately, the UKF is not only computationally more expensive (it requires $2 n+1$ sigma points at each iteration, although there are other approaches that require only $n+2$ sigma points [5]), but it might end up overestimating the final vehicle and map covariances, producing data association errors in the long run. This can be appreciated in the error peaks near $t=90$ secs. in Figures $2 \mathrm{a}-\mathrm{b}$, and in the lower part in Figure 3b. Nearest Neighbor $\chi^{2}$ compatibility tests are used for data association. We believe that the overestimation of covariances is what makes this test to fail when using the full UKF.

By computing the vehicle priors using the Unscented Transformation, the estimated vehicle localization error is smaller during the entire run than the error computed using the EKF; and the computed covariances are quite similar for both algorithms. However, there is a significant advantage at using the UT vs. the EKF: when there are no measurements present, the nonlinear transformation of the vehicle estimates makes a good job at keeping the vehicle location closer to the desired vehicle path. This can be seen around coordinates $(15,5)$ in the three plots pertaining the final vehicle path in Figure 3.

\section{CONCLUSION}

The Unscented Transformation allows a better nonlinear mean and variance estimation than the Extended Kalman Filter. There is no need however in using the Unscented Transformation for the entire vehicle-map state, given the linearity in the map part of the model.

Large underestimation errors in the calculation of the covariance priors can be made when the full state vector is used in the computation of the sigma point set for the Unscented Transformation in SLAM. This is because the more landmarks are added to the map, the larger the number of sigma points in the set that get projected to the vehicle mean in the vehicle localization hyperplane; and the remaining sigma points need to be scaled up to preserve both the mean and covariance.

By applying the Unscented Transformation only to the vehicle states we get more accurate covariance estimates, and a more computationally efficient nonlinear transformation of the means and variances in SLAM. In the presented approach, the a posteriori state estimation is made using a fully observable EKF step, thus preserving the same computational complexity as the EKF with sequential innovation.

\section{APPENDIX I Sigma POINTS}

A set of $p+1$ sigma points $\mathcal{S}=\left\{\mathcal{X}^{i}, W^{i}\right\}$ are deterministically chosen to satisfy a condition set of the form

$$
\mathbf{g}(\mathcal{S}, p(\mathbf{x}))=\mathbf{0}
$$

where $p(\mathbf{x})$ is the $p d f$ of $\mathbf{x}$, not necessarily Gaussian, and $\mathbf{g}(\cdot, \cdot)$ determines the information that should be captured about $\mathbf{x}$. For example, in our Gaussian case, to match the mean

$$
g_{1}=\sum_{i=0}^{p} W^{i} \mathcal{X}^{i}-\mathbf{x}_{k \mid k}
$$

the covariance

$$
g_{2}=\sum_{i=0}^{p} W^{i}\left(\mathcal{X}^{i}-\mathbf{x}_{k \mid k}\right)\left(\mathcal{X}^{i}-\mathbf{x}_{k \mid k}\right)^{\top}-\mathbf{P}_{k \mid k}
$$

and skew

$$
g_{3}=\sum_{i=n}^{p} W^{i}\left(\mathcal{X}^{i}-\mathbf{x}_{k \mid k}\right)^{3} .
$$

One set of points that satisfies such conditions consists in the following symmetrically-distributed set of points [6]:

$$
\begin{aligned}
\mathcal{X}_{k \mid k}^{0} & =\mathbf{x}_{k \mid k} \\
W^{0} & =\frac{\kappa}{n+\kappa} \\
\mathcal{X}_{k \mid k}^{i} & =\mathbf{x}_{k \mid k}+\left(\sqrt{(n+\kappa) \mathbf{P}_{k \mid k}}\right)_{i} \\
\mathcal{X}_{k \mid k}^{i+n} & =\mathbf{x}_{k \mid k}-\left(\sqrt{(n+\kappa) \mathbf{P}_{k \mid k}}\right)_{i} \\
W^{i} & =W^{i+n}=\frac{1}{2(n+\kappa)}
\end{aligned}
$$

where $\left(\sqrt{(n+\kappa) \mathbf{P}_{k \mid k}}\right)_{i}$ is the $i$ th row of the Cholesky decomposition $\mathbf{A}, n \mathbf{P}=\mathbf{A}^{\top} \mathbf{A}$, and $W^{i}$ is the weight associated 
with the $i$ th point. The term $\kappa$ is used to scale the third and higher order terms of this set, and $n$ is the augmented state space dimension (states plus noises).

\section{APPENDIX II}

\section{Nonlinear Vehicle AND Measurement Models}

The vehicle used in our simulations is a pick up truck [9], and is controlled by a velocity $v_{r}$ and a steering angle $\alpha$. The process model used to predict the trajectory of the center of the back axle is given by

$$
\left[\begin{array}{l}
x_{r, k+1} \\
y_{r, k+1} \\
\theta_{r, k+1}
\end{array}\right]=\left[\begin{array}{c}
x_{r, k}+\tau\left(v_{r, k} \cos \theta_{r, k}+v_{x, k}\right) \\
x_{r, k}+\tau\left(v_{r, k} \sin \theta_{r, k}+v_{y, k}\right) \\
\theta_{r, k}+\tau\left(\frac{v_{r, k}}{L} \tan \alpha_{k}+v_{\theta, k}\right)
\end{array}\right]
$$

where $L$ is the distance between wheel axles, $\tau$ is the time constant, and $v_{x}, v_{y}, v_{\theta}$ are zero mean Gaussian model noises.

The observation model is

$$
\left[\begin{array}{c}
z_{r, k}^{i} \\
z_{\beta, k}^{i}
\end{array}\right]=\left[\begin{array}{c}
\sqrt{\left(x_{f}^{i}-x_{r, k}\right)^{2}+\left(y_{f}^{i}-y_{r, k}\right)^{2}}+w_{r, k} \\
\tan ^{-1}\left(\frac{\left(y_{f}^{i}-y_{r, k}\right)}{\left(x_{f}^{i}-x_{r, k}\right)}\right)-\theta_{r, k}+\frac{\pi}{2}+w_{\beta, k}
\end{array}\right]
$$

with $z_{r}^{i}$ and $z_{\beta}^{i}$ the distance and bearing of an observed trunk tree with respect to the vehicle pose. $x_{f}^{i}$ and $x_{f}^{i}$ are the absolute coordinates of such landmark, and $i$ is used for the labeling of landmarks. $i=0$ indicates an anchor feature not under estimation in order to guarantee full observability. $w_{r}$ and $w_{\beta}$ are zero mean Gaussian measurement noises.

\section{REFERENCES}

[1] R. C. Smith and P. Cheeseman, "On the representation and estimation of spatial uncertainty," Int. J. Robot. Res., vol. 5, no. 4, pp. 56-68, 1986.

[2] M. W. M. G. Dissanayake, P. Newman, S. Clark, H. F. Durrant-Whyte, and M. Csorba, "A solution to the simultaneous localization and map building (SLAM) problem,” IEEE Trans. Robot. Automat., vol. 17, no. 3, pp. 229-241, Jun. 2001.

[3] S. Thrun, Y. Liu, D. Koller, A. Y. Ng, Z. Ghahramani, and H. DurrantWhyte, "Simultaneous localization and mapping with sparse extended information filters," Int. J. Robot. Res., vol. 23, no. 7-8, pp. 693-716, Jul. 2004.

[4] S. J. Julier and J. K. Uhlmann, "A counter example to the theory of simultaneous localization and map building," in Proc. IEEE Int. Conf. Robot. Automat., Seoul, May 2001, pp. 4238-4243.

[5] S. J. Julier, "The spherical simplex unscented transformation," in Proc. American Control Conf., Denver, Jun. 2003.

[6] S. J. Julier and J. K. Uhlmann, "Unscented filtering and nonlinear estimation," Proc. IEEE, vol. 92, no. 3, pp. 401-422, Mar. 2004.

[7] _ "A new extension of the Kalman filter to nonlinear systems," in Proc. 11th SPIE Int. Sym. Aerospace/Defense Sensing, Simulation, Controls, I. Kadar, Ed. Orlando: SPIE, Apr. 1997, pp. 182-193.

[8] S. Julier, J. Uhlmann, and H. F. Durrant-Whyte, "A new method for the nonlinear transformation of means and covariances in filters and estimators," IEEE Trans. Automat. Contr., vol. 45, no. 3, pp. 477-482, Mar. 2000.

[9] E. Nebot, J. Guivant, and J. Nieto, "ACFR, experimental outdoor dataset," 2002. [Online]. Available: http://www.acfr.usyd. edu.au/homepages/academic/enebot/dataset.htm

[10] J. E. Guivant and E. M. Nieto, "Optimization of simultaneous localization and map-builidng algorithm for real-time implementation," IEEE Trans. Robot. Automat., vol. 17, no. 3, pp. 242-257, Jun. 2001.

[11] J. Andrade-Cetto and A. Sanfeliu, "The effects of partial observability in SLAM," in Proc. IEEE Int. Conf. Robot. Automat., New Orleans, Apr. 2004, pp. 397-402.

[12] T. Vidal-Calleja, J. Andrade-Cetto, and A. Sanfeliu, "Estimator stability analysis in SLAM," in Proc. 5th IFAC/EURON Sym. Intell. Auton. Vehicles, Lisbon, Jul. 2004.

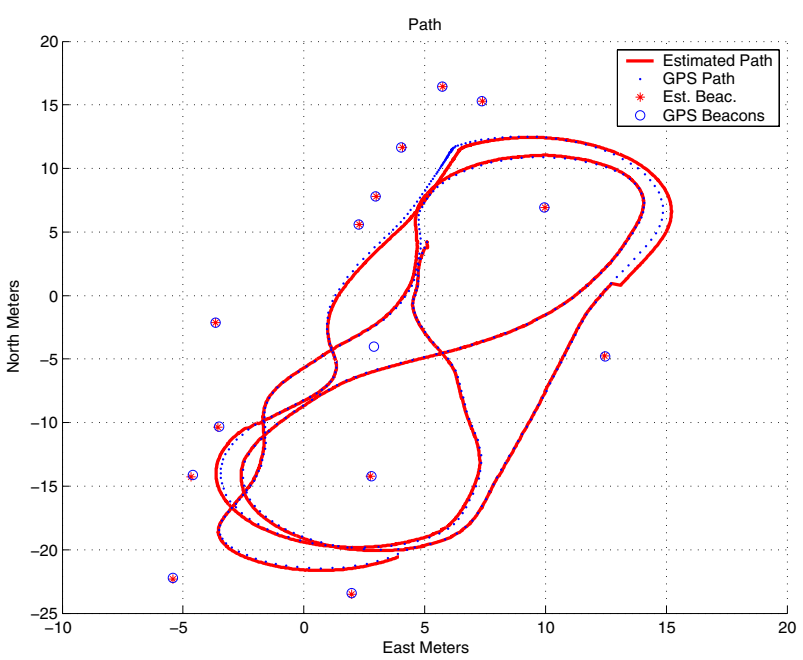

(a) EKF Final map

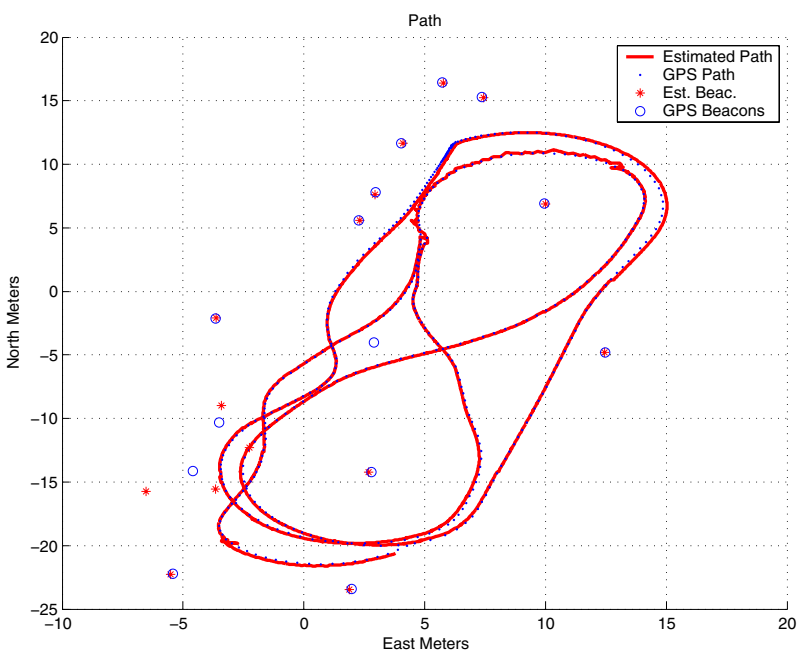

(b) UKF Final map

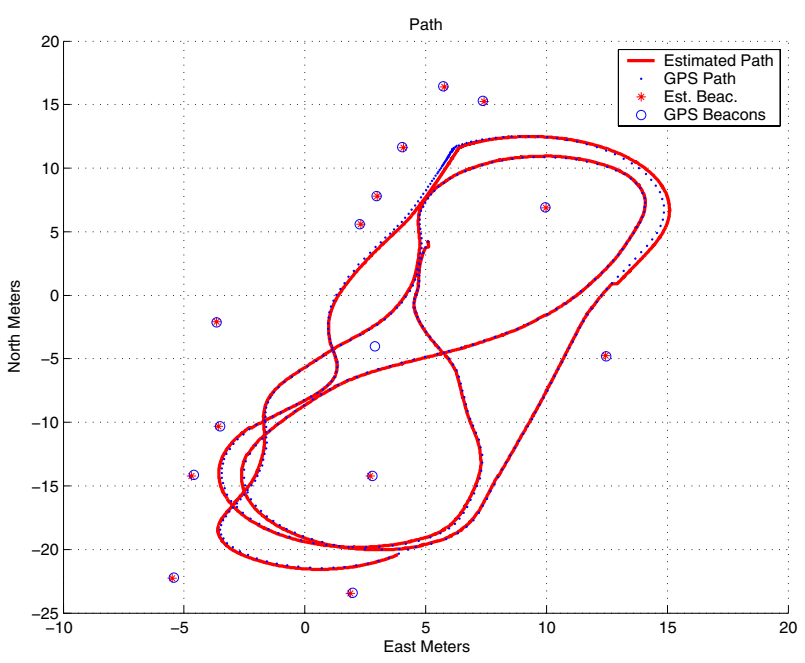

(c) UT Final map

Fig. 3. Comparison of the Extended Kalman Filter, the Unscented Kalman Filter, and Unscented Transformation of Vehicle States only on the Car Park dataset from the University of Sydney. Final vehicle path. 